\title{
On Certain Subclass of Analytic Functions with Fixed Point
}

\author{
T. Al-Hawary, ${ }^{1}$ B. A. Frasin, ${ }^{2}$ and M. Darus ${ }^{1}$ \\ ${ }^{1}$ School of Mathematical Sciences, Faculty of Science and Technology, Universiti Kebangsaan Malaysia, \\ 43600 Bangi, Selangor, Malaysia \\ ${ }^{2}$ Department of Mathematics, Faculty of Science, Al al-Bayt University, Mafraq 130095, Jordan
}

Correspondence should be addressed to M. Darus; maslina@ukm.my

Received 14 October 2012; Revised 27 December 2012; Accepted 27 December 2012

Academic Editor: Yongkun Li

Copyright (C) 2013 T. Al-Hawary et al. This is an open access article distributed under the Creative Commons Attribution License, which permits unrestricted use, distribution, and reproduction in any medium, provided the original work is properly cited.

A new certain differential operator $T_{\alpha, \beta, \delta, \lambda}^{m} f(z)$ and a subclass $S_{m, w}^{*}(\alpha, \beta, \gamma, \delta, \lambda)$ are introduced for functions of the form $f(z)=$ $(z-w)-\sum_{n=2}^{\infty} a_{n}(z-w)^{n}$ which are univalent in the unit disc $U=\{z \in \mathbb{C}:|z|<1\}$. In this paper, we obtain coefficient inequalities, distortion theorem, closure theorems, and class preserving integral operators of functions belonging to the class $S_{m, w}^{*}(\alpha, \beta, \gamma, \delta, \lambda)$.

\section{Introduction}

Let $H(U)$ be the set of functions which are regular in the unit $\operatorname{disc} U=\{z \in \mathbb{C}:|z|<1\}, A=\{f \in H(U): f(0)=$ $\left.f^{\prime}(0)-1=0\right\}$ and $S=\{f \in A: f$ is univalent in $U\}$.

We recall here the definitions of the well-known classes of starlike and convex functions:

$$
\begin{gathered}
S^{*}=\left\{f \in A: \operatorname{Re}\left(\frac{z f^{\prime}(z)}{f(z)}\right)>0, z \in U\right\}, \\
S^{c}=\left\{f \in A: \operatorname{Re}\left(1+\frac{z f^{\prime \prime}(z)}{f^{\prime}(z)}\right)>0, z \in U\right\} .
\end{gathered}
$$

Let $w$ be a fixed point in $U$ and $A(w)=\{f \in H(U)$ : $\left.f(w)=f^{\prime}(w)-1=0\right\}$. classes:

In [1], Kanas and Rønning introduced the following

$$
\begin{gathered}
S_{w}=\{f \in A(w): f \text { is univalent in } U\}, \\
S T_{w}=S_{w}^{*}=\left\{f \in S_{w}: \operatorname{Re}\left(\frac{(z-w) f^{\prime}(z)}{f(z)}\right)>0, z \in U\right\}, \\
S V_{w}=S_{w}^{c}=\left\{f \in S_{w}: 1+\operatorname{Re}\left(\frac{(z-w) f^{\prime \prime}(z)}{f^{\prime}(z)}\right)>0, z \in U\right\} .
\end{gathered}
$$

These classes are extensively studied by Acu and Owa [2].
The class $S_{w}^{*}$ is defined by geometric property that the image of any circular arc centered at $w$ is starlike with respect to $f(w)$ and the corresponding class $S_{w}^{c}$ is defined by the property that the image of any circular arc centered at $w$ is convex. We observe that the definitions are somewhat similar to the ones for uniformly starlike and convex functions introduced by Goodman in $[3,4]$, except that in this case the point $w$ is fixed.

In fact, several subclasses of $S$ have been introduced by a fixed geometric property of the image domain. It is interesting to note that these subclasses play an important role in other branches of mathematics. For example, starlike functions and convex functions play an important role in the solution of certain differential equations (see Robertson [5], Saitoh [6], Owa et al. [7], Reade and Silverman [8], and Sokół and Wiśniowska-Wajnryb [9]). One of the important problems in geometric function theory are the extremal problems, which pose an effective method for establishing the existence of analytic functions with certain natural properties. Extremal problems (supremum $\operatorname{Re}\{f\}$ ) play an important role in geometric function theory, for finding sharp estimates, coefficient bounds, and an extremal function. The results we obtained here may have potential application in other branches of mathematics, both pure and applied. For example the extremal problems are closely connected to Hele-Shaw flow of fluid mechanics [10], exterior inverse problems in potential theory, and many others. 
Now let us begin with our definitions as follows.

The function $f(z)$ in $S_{w}$ is said to be starlike functions of order $\gamma$ if and only if

$$
\operatorname{Re}\left\{\frac{(z-w) f^{\prime}(z)}{f(z)}\right\}>\gamma \quad(z \in U)
$$

for some $\gamma(0 \leq \gamma<1)$. We denote by $S T_{w}(\gamma)$ the class of all starlike functions of order $\gamma$. Similarly, a function $f(z)$ in $S_{w}$ is said to be convex of order $\gamma$ if and only if

$$
\operatorname{Re}\left\{1+\frac{(z-w) f^{\prime \prime}(z)}{f^{\prime}(z)}\right\}>\gamma \quad(z \in U)
$$

for some $\gamma(0 \leq \gamma<1)$. We denote by $S V_{w}(\gamma)$ the class of all convex functions of order $\gamma$.

It is easy to see that $S_{w}$ denoting the subclass of $A(w)$ has the series of expansion:

$$
f(z)=(z-w)+\sum_{n=2}^{\infty} a_{n}(z-w)^{n} .
$$

For the function $f(z)$ in the class $S_{w}$, we define the following new differential operator:

$$
\begin{aligned}
T^{0} f(z)=f(z) & \\
T_{\alpha, \beta, \delta, \lambda}^{1} f(z)= & (1-\beta(\lambda-\alpha)) f(z) \\
& +\beta(\lambda-\alpha)(z-w) f^{\prime}(z)+\delta(z-w)^{2} f^{\prime \prime}(z), \\
T_{\alpha, \beta, \delta, \lambda}^{2} f(z)= & (1-\beta(\lambda-\alpha))\left(T_{\alpha, \beta, \delta, \lambda}^{1} f(z)\right) \\
& +\beta(\lambda-\alpha)(z-w)\left(T_{\alpha, \beta, \delta, \lambda}^{1} f(z)\right)^{\prime} \\
& +\delta(z-w)^{2}\left(T_{\alpha, \beta, \delta, \lambda}^{1} f(z)\right)^{\prime \prime},
\end{aligned}
$$

and for $m=1,2,3, \ldots$.

$$
\begin{aligned}
T_{\alpha, \beta, \delta, \lambda}^{m} f(z)= & (1-\beta(\lambda-\alpha))\left(T_{\alpha, \beta, \delta, \lambda}^{m-1} f(z)\right) \\
& +\beta(\lambda-\alpha)(z-w)\left(T_{\alpha, \beta, \delta, \lambda}^{m-1} f(z)\right)^{\prime} \\
& +\delta(z-w)^{2}\left(T_{\alpha, \beta, \delta, \lambda}^{m-1} f(z)\right)^{\prime \prime} \\
= & (z-w)+\sum_{n=2}^{\infty}[1+(n-1)(\beta(\lambda-\alpha)+n \delta)]^{m} \\
& \times a_{n}(z-w)^{n},
\end{aligned}
$$

for $\alpha \geq 0, \beta \geq 0, \delta \geq 0, \lambda \geq 0$, and $m \in \mathbb{N}_{0}=\mathbb{N} \cup\{0\}$.

It easily verified from (7) that

$$
\begin{aligned}
\beta(\lambda-\alpha)(z-w)\left(T_{\alpha, \beta, \delta, \lambda}^{m} f(z)\right)^{\prime} \\
=T_{\alpha, \beta, \delta, \lambda}^{m+1} f(z)-(1-\beta(\lambda-\alpha)) T_{\alpha, \beta, \delta, \lambda}^{m} f(z) \\
\quad-\delta(z-w)^{2}\left(T_{\alpha, \beta, \delta, \lambda}^{m} f(z)\right)^{\prime \prime} .
\end{aligned}
$$

Remark 1. (i) When $w=\delta=0$, we have the operator introduced and studied by Darus and Ibrahim (see [11]).

(ii) When $w=\alpha=\delta=0$ and $\beta=1$, we have the operator introduced and studied by Al-Oboudi (see [12]).

(iii) When $\alpha=\delta=0$ and $\lambda=\beta=1$, we have the operator introduced and studied by Acu and Owa (see [2]).

(iv) And when $\alpha=\delta=w=0$ and $\lambda=\beta=1$, we have the operator introduced and studied by Sălăgean (see [13]).

With the help of the differential operator $T_{\alpha, \beta, \delta, \lambda}^{m}$, we define the class $S_{m, w}(\alpha, \beta, \gamma, \delta, \lambda)$ as follows.

Definition 2. A function $f(z) \in S_{w}$ is said to be a member of the class $S_{m, w}(\alpha, \beta, \gamma, \delta, \lambda)$ if it satisfies

$$
\begin{aligned}
& \left|\frac{(z-w)\left(T_{\alpha, \beta, \delta, \lambda}^{m} f(z)\right)^{\prime}}{T_{\alpha, \beta, \delta, \lambda}^{m} f(z)}-1\right| \\
& \quad<\left|\frac{(z-w)\left(T_{\alpha, \beta, \delta, \lambda}^{m} f(z)\right)^{\prime}}{T_{\alpha, \beta, \delta, \lambda}^{m} f(z)}+1-2 \gamma\right|
\end{aligned}
$$

for some $0 \leq \gamma<1, \alpha \geq 0, \beta \geq 0, \delta \geq 0, \lambda \geq 0$, and $m \in \mathbb{N}_{0}$ and for all $z \in U$.

It is easy to check that $S_{0,0}(0,1, \gamma, 0,1)$ is the class of starlike functions of order $\gamma$ and $S_{0,0}(0,1,0,0,1)$ gives the all class of starlike functions.

Let $T_{w}$ denote the subclass of $A(w)$ consisting of functions of the following form:

$$
f(z)=(z-w)-\sum_{n=2}^{\infty} a_{n}(z-w)^{n}, \quad a_{n} \geq 0 .
$$

Further, we define the class $S_{m, w}^{*}(\alpha, \beta, \gamma, \delta, \lambda)$ by

$$
S_{m, w}^{*}(\alpha, \beta, \gamma, \delta, \lambda)=S_{m, w}(\alpha, \beta, \gamma, \delta, \lambda) \cap T_{w} .
$$

In this paper, coefficient inequalities, distortion theorem, and closure theorems of functions belonging to the class $S_{m, w}^{*}(\alpha, \beta, \gamma, \delta, \lambda)$ are obtained. Finally, the class preserving integral operators of the form

$$
F(z)=\frac{c+1}{(z-w)^{c}} \int_{w}^{z}(t-w)^{c-1} f(t) d t \quad(c>-1)
$$

for the class $S_{m, w}^{*}(\alpha, \beta, \gamma, \delta, \lambda)$ is considered.

\section{Coefficient Inequalities}

Our first result provides a sufficient condition for a function, regular in $U$, to be in $S_{m, w}^{*}(\alpha, \beta, \gamma, \delta, \lambda)$.

Theorem 3. A function $f(z)$ being defined by (10) is in $S_{m, w}^{*}(\alpha, \beta, \gamma, \delta, \lambda)$ if and only if

$$
\begin{array}{r}
\sum_{n=2}^{\infty}(n-\gamma)[1+(n-1) \beta((\lambda-\alpha)+n \delta)]^{m} a_{n} \leq 1-\gamma \\
\left(m \in \mathbb{N}_{0}\right),
\end{array}
$$


where $0 \leq \gamma<1$. The result (13) is sharp for functions of the following form:

$$
\begin{aligned}
& f_{n}(z)=(z-w) \\
&-\frac{1-\gamma}{(n-\gamma)[1+(n-1) \beta((\lambda-\alpha)+n \delta)]^{m}}(z-w)^{n} \\
& \quad\left(n \geq 2 ; m \in \mathbb{N}_{0}\right) .
\end{aligned}
$$

Proof. Suppose that (13) holds true for $0 \leq \gamma<1$. consider the expression

$$
\begin{aligned}
M\left(f, f^{\prime}\right) & \\
= & \left|(z-w)\left(T_{\alpha, \beta, \delta, \lambda}^{m} f(z)\right)^{\prime}-\left(T_{\alpha, \beta, \delta, \lambda}^{m} f(z)\right)\right| \\
& -\left|(z-w)\left(T_{\alpha, \beta, \delta, \lambda}^{m} f(z)\right)^{\prime}+(1-2 \gamma)\left(T_{\alpha, \beta, \delta, \lambda}^{m} f(z)\right)\right| .
\end{aligned}
$$

Then for $|z-w|=r<1$, we have

$$
\begin{aligned}
& M\left(f, f^{\prime}\right) \\
& =\mid \sum_{n=2}^{\infty}(n-1)[1+(n-1) \beta((\lambda-\alpha)+n \delta)]^{m} \\
& \times a_{n}(z-w)^{n} \\
& -\mid 2(1-\gamma)(z-w) \\
& -\sum_{n=2}^{\infty}(n-2 \gamma+1)[1+(n-1) \beta((\lambda-\alpha)+n \delta)]^{m} \\
& \times a_{n}(z-w)^{n} \mid, \\
& M\left(f, f^{\prime}\right) \\
& \leq \sum_{n=2}^{\infty}(n-1) \\
& \times[1+(n-1) \beta((\lambda-\alpha)+n \delta)]^{m} a_{n} r^{n} \\
& -2(1-\gamma)+\sum_{n=2}^{\infty}(n+2 \gamma-1) \\
& \times[1+\lambda(n+p-2)]^{m} a_{n} r^{n} \\
& \leq \sum_{n=2}^{\infty} 2(n-\gamma)[1+(n-1) \beta((\lambda-\alpha)+n \delta)]^{m} \\
& \times a_{n}-2(1-\gamma) \leq 0 .
\end{aligned}
$$

So that $f(z) \in S_{m, w}^{*}(\alpha, \beta, \gamma, \delta, \lambda)$.
For the converse, assume that

$$
\begin{gathered}
\left|\frac{(z-w)\left(T_{\alpha, \beta, \delta, \lambda}^{m} f(z)\right)^{\prime}}{T_{\alpha, \beta, \delta, \lambda}^{m} f(z)}-1\right| \\
/\left|\frac{(z-w)\left(T_{\alpha, \beta, \delta, \lambda}^{m} f(z)\right)^{\prime}}{T_{\alpha, \beta, \delta, \lambda}^{m} f(z)}+1-2 \gamma\right| \\
=\mid\left(-\sum_{n=2}^{\infty}(n-1)[1+(n-1) \beta((\lambda-\alpha)+n \delta)]^{m}\right. \\
\left.\times a_{n}(z-w)^{n}\right)
\end{gathered}
$$$$
\times(2(1-\gamma)(z-w)
$$

$$
\begin{gathered}
-\sum_{n=2}^{\infty}(n-2 \gamma+1)[1+(n-1) \beta((\lambda-\alpha)+n \delta)]^{m} \\
\left.\times a_{n}(z-w)^{n}\right)^{-1}
\end{gathered}
$$

$<1$.

Since $\operatorname{Re}(z-w) \leq|z-w|$ for all $(z-w)$, it follows from (17) that

$$
\begin{gathered}
\operatorname{Re}\left\{\left(\sum_{n=2}^{\infty}(n-1)[1+(n-1) \beta((\lambda-\alpha)+n \delta)]^{m}\right.\right. \\
\left.\times a_{n}(z-w)^{n}\right) \\
\times(2(1-\gamma)(z-w) \\
-\sum_{n=2}^{\infty}(n-2 \gamma+1)[1+(n-1) \beta((\lambda-\alpha)+n \delta)]^{m} \\
\left.\left.\times a_{n}(z-w)^{n}\right)^{-1}\right\}
\end{gathered}
$$

$<1$.

Choose values of $(z-w)$ on the real axis so that $(z-w)\left(T_{\alpha, \beta, \delta, \lambda}^{m} f(z)\right)^{\prime} / T_{\alpha, \beta, \delta, \lambda}^{m} f(z)$ is real. Upon clearing the 
denominator in (18) and letting $r \rightarrow 1^{-}$through real values, we obtain

$$
\begin{aligned}
\sum_{n=2}^{\infty}(n-1)[1+(n-1) \beta((\lambda-\alpha)+n \delta)]^{m} a_{n} \\
\quad<2(1-\gamma) \\
\quad-\sum_{n=2}^{\infty}(n-2 \gamma+1)[1+(n-1) \beta((\lambda-\alpha)+n \delta)]^{m} a_{n} .
\end{aligned}
$$

This gives the required condition. Hence the theorem follows.

Corollary 4. Let the function $f(z)$ be defined by (10) and $f(z) \in S_{w}$. If $f \in S_{m, w}^{*}(\alpha, \beta, \gamma, \delta, \lambda)$, then

$$
a_{n} \leq \frac{1-\gamma}{(n-\gamma)[1+(n-1) \beta((\lambda-\alpha)+n \delta)]^{m}}, \quad n \geq 2 .
$$

The result (20) is sharp for functions $f_{n}(z)$ given by (14).

\section{Distortion Theorem}

A distortion property for functions in the class $f \in$ $S_{m, w}^{*}(\alpha, \beta, \gamma, \delta, \lambda)$ is contained in

Theorem 5. Let the function $f(z)$ one has defined by (10) be in the class $f \in S_{m, w}^{*}(\alpha, \beta, \gamma, \delta, \lambda)$. Then for $|z-w|=r<1$, we have

$$
\begin{aligned}
r- & \frac{1-\gamma}{(2-\gamma)[1+\beta((\lambda-\alpha)+2 \delta)]^{m}} r^{2} \\
& \leq|f(z)| \\
& \leq r+\frac{1-\gamma}{(2-\gamma)[1+\beta((\lambda-\alpha)+2 \delta)]^{m}} r^{2}, \\
1- & \frac{2(1-\gamma)}{(2-\gamma)[1+\beta((\lambda-\alpha)+2 \delta)]^{m}} r \\
\leq & \left|f^{\prime}(z)\right| \\
& \leq 1+\frac{2(1-\gamma)}{(2-\gamma)[1+\beta((\lambda-\alpha)+2 \delta)]^{m}} r .
\end{aligned}
$$

Proof. Since $f \in S_{m, w}^{*}(\alpha, \beta, \gamma, \delta, \lambda)$, Theorem 3 readily yields the inequality

$$
\sum_{n=2}^{\infty} a_{n} \leq \frac{1-\gamma}{(2-\gamma)[1+\beta((\lambda-\alpha)+2 \delta)]^{m}} .
$$

Thus, for $|z-w|=r<1$, and making use of (22), we have

$$
\begin{aligned}
|f(z)| & \leq|z-w|+\sum_{n=2}^{\infty} a_{n}|z-w|^{n} \\
& \leq r+r^{2} \sum_{n=2}^{\infty} a_{n} \\
& \leq r+\frac{1-\gamma}{(2-\gamma)[1+\beta((\lambda-\alpha)+2 \delta)]^{m}} r^{2}, \\
|f(z)| & \geq|z-w|-\sum_{n=2}^{\infty} a_{n}|z-w|^{n}
\end{aligned}
$$

$$
\begin{aligned}
& \geq r-r^{2} \sum_{n=2}^{\infty} a_{n} \\
& \geq r-\frac{1-\gamma}{(2-\gamma)[1+\beta((\lambda-\alpha)+2 \delta)]^{m}} r^{2} .
\end{aligned}
$$

Also from Theorem 3, it follows that

$$
\begin{aligned}
& \frac{(2-\gamma)[1+\beta((\lambda-\alpha)+2 \delta)]^{m}}{2} \sum_{n=2}^{\infty} n a_{n} \\
& \leq \sum_{n=2}^{\infty}(n-\gamma)[1+(n-1) \beta((\lambda-\alpha)+n \delta)]^{m} a_{n} \\
& \quad \leq 1-\gamma .
\end{aligned}
$$

Hence

$$
\begin{aligned}
& \left|f^{\prime}(z)\right| \\
& \quad \leq 1+\sum_{n=2}^{\infty} n a_{n}|z-w|^{n-1} \\
& \quad \leq 1+r \sum_{n=2}^{\infty} n a_{n} \\
& \quad \leq 1+\frac{2(1-\gamma)}{(2-\gamma)[1+\beta((\lambda-\alpha)+2 \delta)]^{m}} r, \\
& \left|f^{\prime}(z)\right| \\
& \quad \geq 1-\sum_{n=2}^{\infty} n a_{n}|z-w|^{n-1} \\
& \quad \geq 1-r \sum_{n=2}^{\infty} n a_{n} \\
& \quad \geq 1-\frac{2(1-\gamma)}{(2-\gamma)[1+\beta((\lambda-\alpha)+2 \delta)]^{m}} r .
\end{aligned}
$$

This completes the proof of Theorem 5 .

\section{Radii of Starlikeness and Convexity}

The radii of starlikeness and convexity for the class $S_{m, w}^{*}(\alpha, \beta, \gamma, \delta, \lambda)$ is given by the following theorems. 
Theorem 6. If the function $f(z)$ defined by (10) is in the class $S_{m, w}^{*}(\alpha, \beta, \gamma, \delta, \lambda)$, then $f(z)$ is of order $\eta(0 \leq \eta<1)$ in $\mid z-$ $w \mid<r_{1}$, where

$$
r_{1}=\inf _{n \geq 2}\left\{\frac{(1-\eta)(n-\gamma)[1+(n-1) \beta((\lambda-\alpha)+n \delta)]^{m}}{(n-\eta)(1-\gamma)}\right\}^{1 / n} .
$$

The result is sharp for the functions $f_{n}(z)$ given by (14).

Proof. It suffices to prove that

$$
\left|\frac{(z-w) f^{\prime}(z)}{f(z)}-1\right| \leq 1-\eta
$$

for $|z-w|<r_{1}$. We have

$$
\begin{aligned}
& \left|\frac{(z-w) f^{\prime}(z)}{f(z)}-1\right| \\
& \quad=\left|\frac{-\sum_{n=2}^{\infty}(n-1) a_{n}(z-w)^{n}}{(z-w)-\sum_{n=2}^{\infty} a_{n}(z-w)^{n}}\right| \\
& \quad \leq \frac{\sum_{n=2}^{\infty}(n-1) a_{n}|z-w|^{n}}{1-\sum_{n=2}^{\infty} a_{n}|z-w|^{n}} .
\end{aligned}
$$

Hence (28) holds true if

$$
\begin{aligned}
& \sum_{n=2}^{\infty}(n-1) a_{n}|z-w|^{n} \\
& \quad \leq(1-\eta)\left(1-\sum_{n=2}^{\infty} a_{n}|z-w|^{n}\right)
\end{aligned}
$$

or

$$
\sum_{n=2}^{\infty} \frac{n-\eta}{1-\eta} a_{n}|z-w|^{n} \leq 1
$$

with the aid of (13), (30) is true if

$$
\begin{aligned}
& \frac{n-\eta}{1-\eta}|z-w|^{n} \\
& \quad \leq \frac{(n-\gamma)[1+(n-1) \beta((\lambda-\alpha)+n \delta)]^{m}}{1-\gamma} .
\end{aligned}
$$

Solving (31) for $|z-w|$, we obtain

$$
\begin{aligned}
& |z-w| \\
& \leq\left\{\frac{(1-\eta)(n-\gamma)[1+(n-1) \beta((\lambda-\alpha)+n \delta)]^{m}}{(n-\eta)(1-\gamma)}\right\}^{1 / n} \\
& (n \geq 2) .
\end{aligned}
$$

Theorem 7. If the function $f(z)$ defined by (10) is in the class $S_{m, w}^{*}(\alpha, \beta, \gamma, \delta, \lambda)$, then $f(z)$ is convex of order $\eta(0 \leq \eta<1)$ in $|z-w|<r_{2}$, where

$$
r_{2}=\inf _{n \geq 2}\left\{\frac{(1-\eta)(n-\gamma)[1+(n-1) \beta((\lambda-\alpha)+n \delta)]^{m}}{n(n+\eta-2)(1-\gamma)}\right\}^{1 /(n-1)}
$$

The result is sharp for the functions $f_{n}(z)$ given by (14).

Proof. By using the technique employed in the proof of Theorem 6, we can show that

$$
\left|\frac{(z-w) f^{\prime \prime}(z)}{f^{\prime}(z)}\right| \leq 1-\eta
$$

for $|z-w|<r_{2}$, with the aid of Theorem 3. Thus we have the assertion of Theorem 7 .

\section{Closure Theorems}

Let the functions $f_{j}(z), j=1,2, \ldots, l$, be defined by

$$
f_{j}(z)=(z-w)-\sum_{n=2}^{\infty} a_{n, j}(z-w)^{n}, \quad\left(a_{n, j} \geq 0\right)
$$

for $z \in U$.

Theorem 8. Let the functions $f_{j}(z)$ be defined by (35) in the class $S_{m, w}^{*}(\alpha, \beta, \gamma, \delta, \lambda)$ for every $j=1,2, \ldots, l$. Then the function $G(z)$ defined by

$$
G(z)=(z-w)-\sum_{n=2}^{\infty} b_{n}(z-w)^{n}, \quad\left(b_{n} \geq 0\right)
$$

is a member of the class $S_{m, w}^{*}(\alpha, \beta, \gamma, \delta, \lambda)$, where

$$
b_{n}=\frac{1}{l} \sum_{j=1}^{l} a_{n, j} \quad(n \geq 2) .
$$

Proof. Since $f_{j}(z) \in S_{m, w}^{*}(\alpha, \beta, \gamma, \delta, \lambda)$, it follows from Theorem 3 that

$$
\sum_{n=2}^{\infty}(n-\gamma)[1+(n-1) \beta((\lambda-\alpha)+n \delta)]^{m} a_{n, j} \leq 1-\gamma
$$

This completes the proof of Theorem 6 . 
for every $j=1,2, \ldots, l$. Hence,

$$
\begin{aligned}
& \sum_{n=2}^{\infty}(n-\gamma)[1+(n-1) \beta((\lambda-\alpha)+n \delta)]^{m} b_{n} \\
& =\sum_{n=2}^{\infty}(n-\gamma)[1+(n-1) \beta((\lambda-\alpha)+n \delta)]^{m} \\
& \quad \times\left\{\frac{1}{l} \sum_{j=1}^{l} a_{n, j}\right\} \\
& =\frac{1}{l} \sum_{j=1}^{l}\left(\sum_{n=2}^{\infty}(n-\gamma)\right. \\
& \left.\quad \times[1+(n-1) \beta((\lambda-\alpha)+n \delta)]^{m} a_{n, j}\right) \\
& \leq \frac{1}{l} \sum_{j=1}^{l}(1-\gamma)=1-\gamma
\end{aligned}
$$

which (in view of Theorem 5) implies that $G(z) \epsilon$ $S_{m, w}^{*}(\alpha, \beta, \gamma, \delta, \lambda)$.

Theorem 9. The class $S_{m, w}^{*}(\alpha, \beta, \gamma, \delta, \lambda)$ is closed under convex linear combination.

Proof. Suppose that the functions $f_{j}(z)(j=1,2)$ defined by (35) be in the class $S_{m, w}^{*}(\alpha, \beta, \gamma, \delta, \lambda)$, it is sufficient to prove that the function

$$
H(z)=\mu f_{1}(z)+(1-\mu) f_{2}(z) \quad(0 \leq \mu \leq 1)
$$

is also in the class $S_{m, w}^{*}(\alpha, \beta, \gamma, \delta, \lambda)$. Since, for $0 \leq \mu \leq 1$,

$$
H(z)=(z-w)-\sum_{n=2}^{\infty}\left\{\mu a_{n, 1}+(1-\mu) a_{n, 2}\right\}(z-w)^{n}
$$

we observe that

$$
\begin{aligned}
& \sum_{n=2}^{\infty}(n-\gamma)[1+(n-1) \beta((\lambda-\alpha)+n \delta)]^{m} \\
& \quad \times\left\{\mu a_{n, 1}+(1-\mu) a_{n, 2}\right\} \\
& =\mu \sum_{n=2}^{\infty}(n-\gamma)[1+(n-1) \beta((\lambda-\alpha)+n \delta)]^{m} a_{n, 1} \\
& \quad+(1-\mu) \sum_{n=2}^{\infty}(n-\gamma) \\
& \quad \times[1+(n-1) \beta((\lambda-\alpha)+n \delta)]^{m} a_{n, 2} \\
& \leq \mu(1-\gamma)+(1-\mu)(1-\gamma)=(1-\gamma) .
\end{aligned}
$$

Hence $H(z) \in S_{m, w}^{*}(\alpha, \beta, \gamma, \delta, \lambda)$. This completes the proof of Theorem 9.
Theorem 10. Let

$$
\begin{aligned}
& f_{0}(z)=z-w, \\
& f_{n}(z)=(z-w) \\
& -\frac{1-\gamma}{(n-\gamma)[1+(n-1) \beta((\lambda-\alpha)+n \delta)]^{m}}(z-w)^{n} \\
& \quad\left(n \geq 2 ; m \in \mathbb{N}_{0}\right) .
\end{aligned}
$$

Then $f(z) \in S_{m, w}^{*}(\alpha, \beta, \gamma, \delta, \lambda)$ if and only if it can be expressed in the form

$$
f(z)=\sum_{n=1}^{\infty} \lambda_{n} f_{n}(z)
$$

where $\lambda_{n} \geq 0$ and $\sum_{n=1}^{\infty} \lambda_{n}=1$.

Proof. Suppose that

$$
f(z)=\sum_{n=1}^{\infty} \lambda_{n} f_{n}(z)
$$

where $\lambda_{n} \geq 0(n \geq 1)$ and $\sum_{n=1}^{\infty} \lambda_{n}=1$. Then

$$
\begin{aligned}
f(z)= & \sum_{n=1}^{\infty} \lambda_{n} f_{n}(z) \\
= & \lambda_{1} f_{1}(z)+\sum_{n=2}^{\infty} \lambda_{n} f_{n}(z) \\
= & (z-w) \\
& -\sum_{n=2}^{\infty} \frac{1-\gamma}{(n-\gamma)[1+(n-1) \beta((\lambda-\alpha)+n \delta)]^{m}} \\
& \times \lambda_{n}(z-w)^{n} .
\end{aligned}
$$

Since

$$
\begin{aligned}
& \sum_{n=2}^{\infty}(n-\gamma)[1+(n-1) \beta((\lambda-\alpha)+n \delta)]^{m} \\
& \cdot \frac{1-\gamma}{(n-\gamma)[1+(n-1) \beta((\lambda-\alpha)+n \delta)]^{m}} \lambda_{n} \\
& \quad=(1-\gamma) \sum_{n=2}^{\infty} \lambda_{n} \\
& \quad=(1-\gamma)\left(1-\lambda_{0}\right) \leq 1-\gamma,
\end{aligned}
$$

it follows from Theorem 3 that the function $f(z) \epsilon$ $S_{m, w}^{*}(\alpha, \beta, \gamma, \delta, \lambda)$. Conversely, let us suppose that $f(z) \in$ $S_{m, w}^{*}(\alpha, \beta, \gamma, \delta, \lambda)$. Since

$$
a_{n} \leq \frac{1-\gamma}{(n-\gamma)[1+(n-1) \beta((\lambda-\alpha)+n \delta)]^{m}}
$$


Setting

$$
\begin{gathered}
\lambda_{n}=\frac{(n-\gamma)[1+(n-1) \beta((\lambda-\alpha)+n \delta)]^{m}}{1-\gamma} a_{n}, \\
\left(n \geq 2 ; m \in \mathbb{N}_{0}\right), \\
\lambda_{1}=1-\sum_{n=2}^{\infty} \lambda_{n},
\end{gathered}
$$

it follows that $f(z)=S_{m, w}^{*}(\alpha, \beta, \gamma, \delta, \lambda)$. This completes the proof of the theorem.

\section{Extreme Points}

Theorem 11. Let

$$
\begin{aligned}
& f_{0}(z)=z-w \\
& f_{n}(z)=(z-w) \\
&-\frac{1-\gamma}{(n-\gamma)[1+(n-1) \beta((\lambda-\alpha)+n \delta)]^{m}} \\
& \times(z-w)^{n} \quad\left(n \geq 2 ; m \in \mathbb{N}_{0}\right) .
\end{aligned}
$$

Then $f(z) \in S_{m, w}^{*}(\alpha, \beta, \gamma, \delta, \lambda)$ if and only if it can be expressed in the form

$$
f(z)=\sum_{n=1}^{\infty} \lambda_{n} f_{n}(z)
$$

where $\lambda_{n} \geq 0$ and $\sum_{n=1}^{\infty} \lambda_{n}=1$.

Proof. Suppose that

$$
f(z)=\sum_{n=1}^{\infty} \lambda_{n} f_{n}(z)
$$

where $\lambda_{n} \geq 0(n \geq 1)$ and $\sum_{n=1}^{\infty} \lambda_{n}=1$. Then

$$
\begin{aligned}
f(z)= & \sum_{n=1}^{\infty} \lambda_{n} f_{n}(z) \\
= & \lambda_{1} f_{1}(z)+\sum_{n=2}^{\infty} \lambda_{n} f_{n}(z) \\
= & (z-w) \\
& -\sum_{n=2}^{\infty} \frac{1-\gamma}{(n-\gamma)[1+(n-1) \beta((\lambda-\alpha)+n \delta)]^{m}} \\
& \quad \times \lambda_{n}(z-w)^{n} .
\end{aligned}
$$

Since

$$
\begin{aligned}
\sum_{n=2}^{\infty}(n-\gamma)[1+(n-1) \beta((\lambda-\alpha)+n \delta)]^{m} \\
\cdot \frac{1-\gamma}{(n-\gamma)[1+(n-1) \beta((\lambda-\alpha)+n \delta)]^{m}} \lambda_{n} \\
=(1-\gamma) \sum_{n=2}^{\infty} \lambda_{n} \\
=(1-\gamma)\left(1-\lambda_{0}\right) \leq 1-\gamma,
\end{aligned}
$$

it follows from Theorem 3 that the function $f(z) \in$ $S_{m, w}^{*}(\alpha, \beta, \gamma, \delta, \lambda)$. Conversely, let us suppose that $f(z) \in$ $S_{m, w}^{*}(\alpha, \beta, \gamma, \delta, \lambda)$. Since

$$
\begin{array}{r}
a_{n} \leq \frac{1-\gamma}{(n-\gamma)[1+(n-1) \beta((\lambda-\alpha)+n \delta)]^{m}} \\
\quad\left(n \geq 2 ; m \in \mathbb{N}_{0}\right) .
\end{array}
$$

Setting

$$
\begin{gathered}
\lambda_{n}=\frac{(n-\gamma)[1+(n-1) \beta((\lambda-\alpha)+n \delta)]^{m}}{1-\gamma} a_{n}, \\
\left(n \geq 2 ; m \in \mathbb{N}_{0}\right), \\
\lambda_{1}=1-\sum_{n=2}^{\infty} \lambda_{n},
\end{gathered}
$$

it follows that $f(z)=S_{m, w}^{*}(\alpha, \beta, \gamma, \delta, \lambda)$. This completes the proof of theorem.

\section{Integral Operators}

Theorem 12. If the function $f(z)$ defined by (10) is in the class $S_{m, w}^{*}(\alpha, \beta, \gamma, \delta, \lambda)$, and let $c$ be a real number such that $c>-1$. Then the function $F(z)$ defined by

$$
F(z)=\frac{c+1}{(z-w)^{c}} \int_{w}^{z}(t-w)^{c-1} f(t) d t
$$

also belongs to the class $S_{m, w}^{*}(\alpha, \beta, \gamma, \delta, \lambda)$.

Proof. From (57), it follows that $F(z)=(z-w)-$ $\sum_{n=2}^{\infty} b_{n}(z-w)^{n}$, where $b_{n}=((c+1) /(c+n)) a_{n}$. Therefore

$$
\begin{aligned}
& \sum_{n=2}^{\infty}(n-\gamma)[1+(n-1) \beta((\lambda-\alpha)+n \delta)]^{m} b_{n} \\
& =\sum_{n=2}^{\infty}(n-\gamma)[1+(n-1) \beta((\lambda-\alpha)+n \delta)]^{m} \\
& \quad \times\left(\frac{c+1}{c+n}\right) a_{n} \\
& \leq \sum_{n=2}^{\infty}(n-\gamma)[1+(n-1) \beta((\lambda-\alpha)+n \delta)]^{m} a_{n} \\
& \leq 1-\gamma,
\end{aligned}
$$


since $f(z) \in S_{m, w}^{*}(\alpha, \beta, \gamma, \delta, \lambda)$. Hence by Theorem $3, F(z) \in$ $S_{m, w}^{*}(\alpha, \beta, \gamma, \delta, \lambda)$.

\section{Acknowledgment}

The work here was fully supported by LRGS/TD/2011/ UKM/ICT/03/02 and UKM-DLP-2011-050.

\section{References}

[1] S. Kanas and F. Rønning, "Uniformly starlike and convex functions and other related classes of univalent functions," Annales Universitatis Mariae Curie-Skłodowska A, vol. 53, pp. 95-105, 1999.

[2] M. Acu and S. Owa, "On a subclass of $n$-starlike functions," International Journal of Mathematics and Mathematical Sciences, vol. 2005, no. 17, pp. 2841-2846, 2005.

[3] A. W. Goodman, "On uniformly starlike functions," Journal of Mathematical Analysis and Applications, vol. 155, no. 2, pp. 364370, 1991.

[4] A. W. Goodman, "On uniformly convex functions," Annales Polonici Mathematici, vol. 56, no. 1, pp. 87-92, 1991.

[5] M. S. Robertson, "Extremal problems for analytic functions with positive real part and applications," Transactions of the American Mathematical Society, vol. 106, pp. 236-253, 1963.

[6] H. Saitoh, "Univalence and starlikeness of solutions of $W$ " + $a W^{\prime}+b W=0$," Annales Universitatis Mariae Curie-Skłodowska A, vol. 53, pp. 209-216, 1999.

[7] S. Owa, H. Saitoh, H. M. Srivastava, and R. Yamakawa, "Geometric properties of solutions of a class of differential equations," Computers \& Mathematics with Applications, vol. 47, no. 10-11, pp. 1689-1696, 2004.

[8] M. O. Reade and H. Silverman, "Radii problems for linear properties of univalent functions," Houston Journal of Mathematics, vol. 17, no. 2, pp. 227-235, 1991.

[9] J. Sokół and A. Wiśniowska-Wajnryb, "On certain problem in the class of $k$-starlike functions," Computers \& Mathematics with Applications, vol. 62, no. 12, pp. 4733-4741, 2011.

[10] P. Curt and D. Fericean, "A special class of univalent functions in Hele-Shaw flow problems," Abstract and Applied Analysis, vol. 2011, Article ID 948236, 10 pages, 2011.

[11] M. Darus and R. W. Ibrahim, "On subclasses for generalized operators of complex order," Far East Journal of Mathematical Sciences, vol. 33, no. 3, pp. 299-308, 2009.

[12] F. M. Al-Oboudi, "On univalent functions defined by a generalized Sălăgean operator," International Journal of Mathematics and Mathematical Sciences, vol. 2004, no. 27, pp. 1429-1436, 2004.

[13] G. Sălăgean, "Subclasses of univalent functions," in Lecture Notes in Mathematics, pp. 362-372, Springer, Heidelberg, Germany, 1983. 


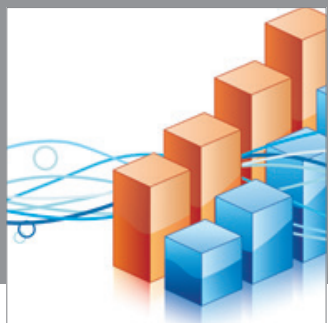

Advances in

Operations Research

mansans

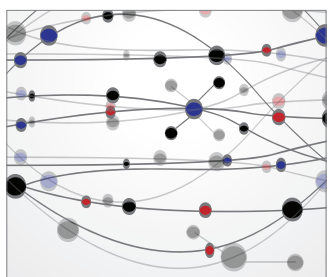

The Scientific World Journal
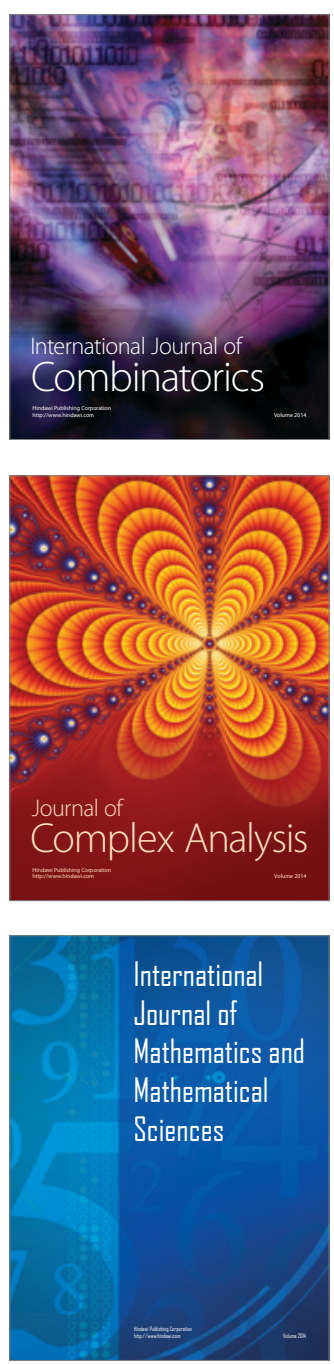
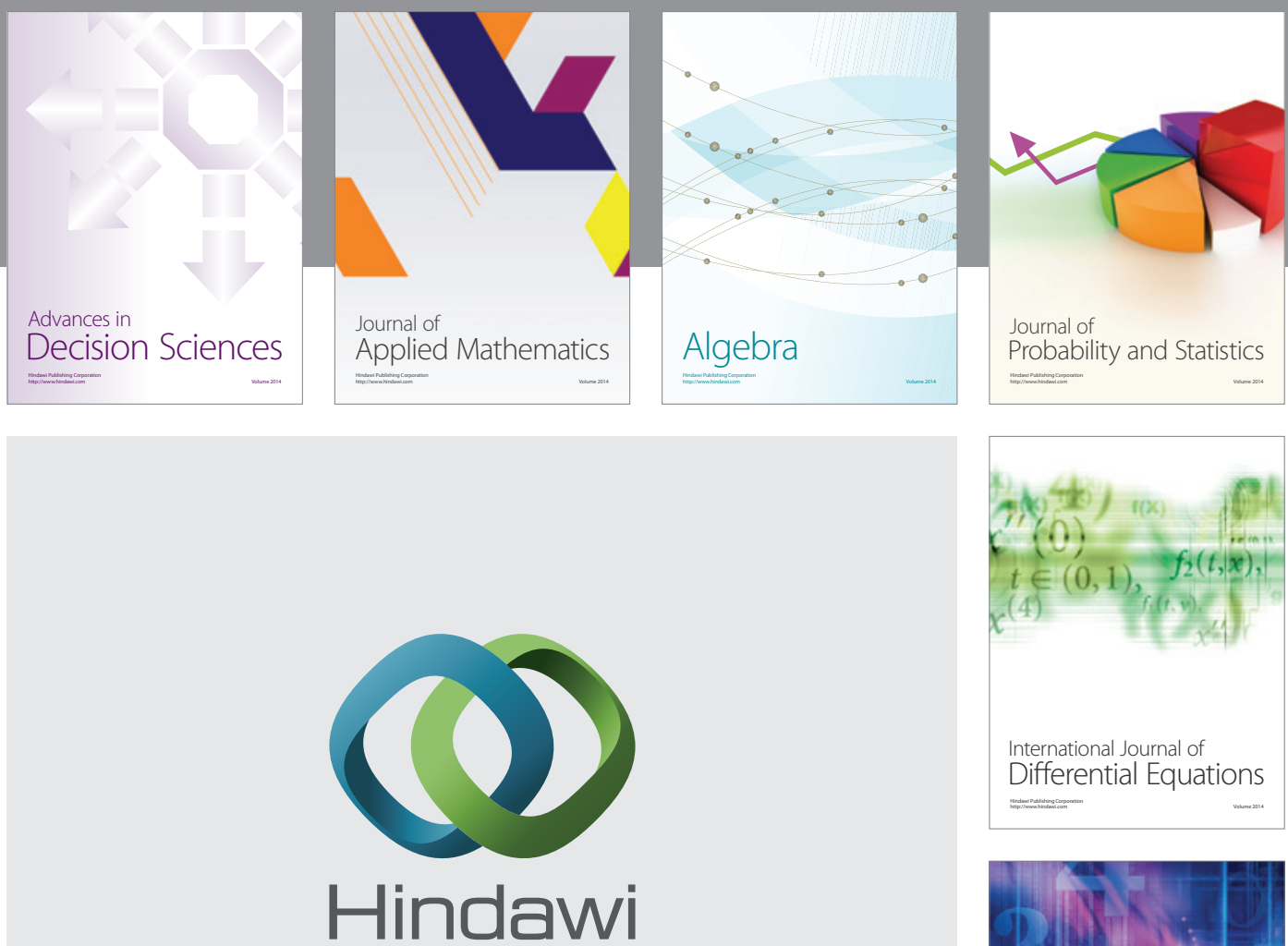

Submit your manuscripts at http://www.hindawi.com
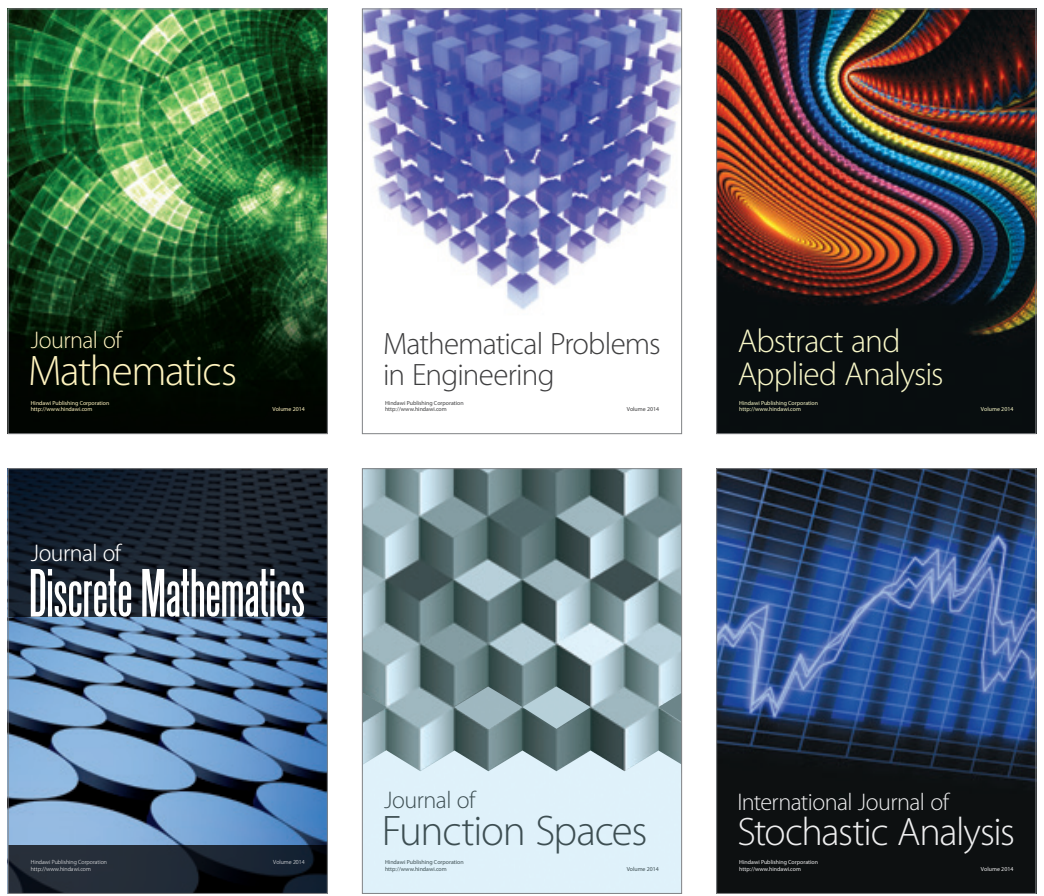

Journal of

Function Spaces

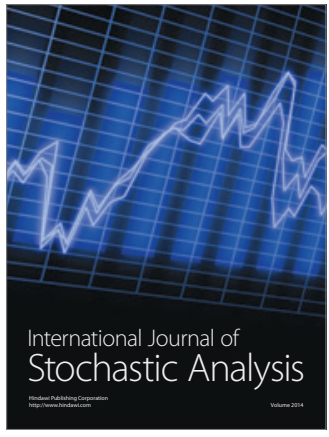

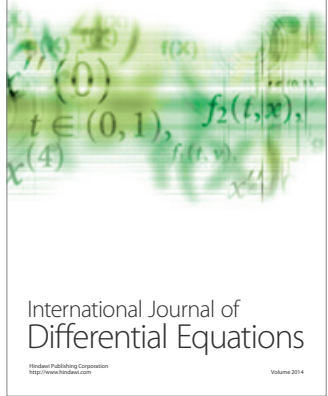
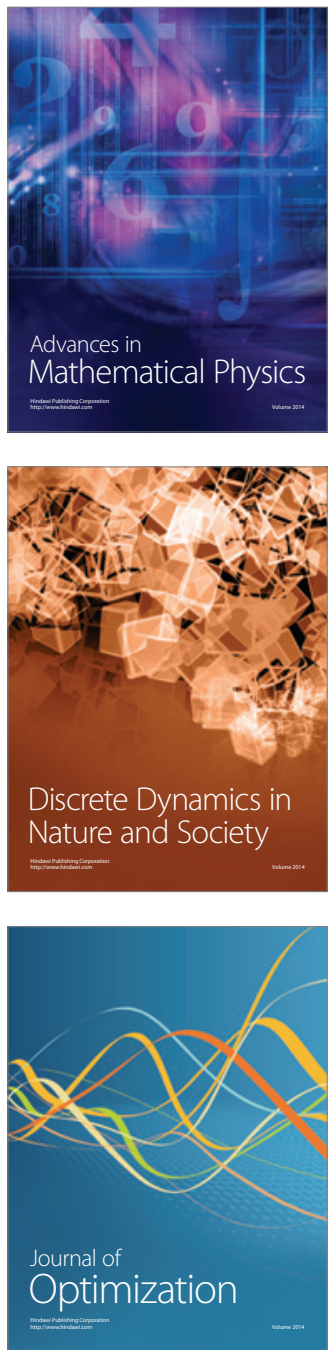